F. Nobakht, A.V. Kamyad, GH. Atazandi, A. Zare / TJMCS Vol .3 No.3 (2011) 346 - 356

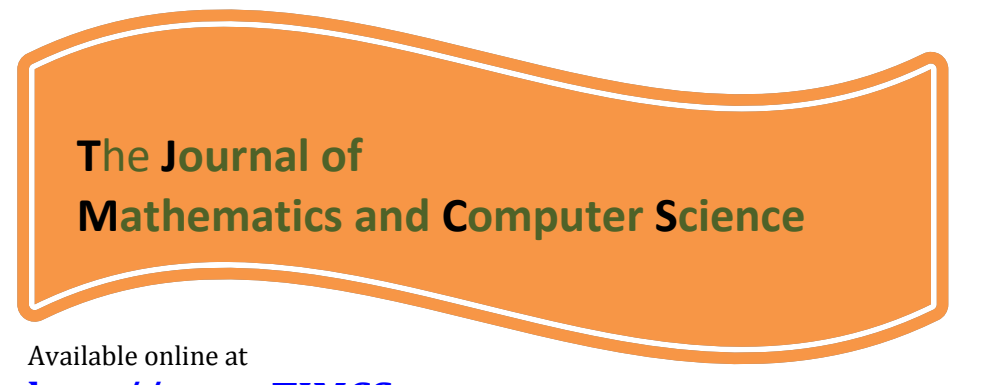

http://www.TJMCS.com

The Journal of Mathematics and Computer Science Vol .3 No.3 (2011) 346 - 356

\title{
Optimal Solution For System of kth-order Fuzzy Differential Equations
}

\author{
F. Nobakht ${ }^{a}$, A.V. Kamyad $^{b}$, GH. Atazandi ${ }^{c}$, A. Zare ${ }^{c}$ \\ ${ }^{a}$ Department of Electrical Engineering, Asrar University of Mashhad , Iran \\ ${ }^{b}$ Department of Mathematics, Ferdowsi University of Mashhad, Iran \\ ${ }^{c}$ Department of Electrical Engineering, Islamic Azad University Branch of Gonabad, Iran \\ Nobakht_fariba7@yahoo.com \\ Zandi_reza7@yahoo.com \\ Asefzare@yahoo.com
}

Received: August 2011, Revised: November 2011

Online Publication: December 2011

\begin{abstract}
To solving fuzzy control problems ,classical method are not usually efficient .In this paper we proposed a new approach for solving this class of problem by linear programming problems(LPP).First we transfer the original problem to a new problem in form of calculus of variations. Then we discretize the new problem and solve it by using LPP packages Finally, efficiency of our approach is confirmed by some numerical example.
\end{abstract}

Keywords: Fuzzy differential equations, AVK method, Numerical method, optimal control.

\section{Introduction}

The topic of Fuzzy Differential Equations(FDEs), has been rapidly growing in recent years. The concept of the fuzzy derivative was first introduced by Chang and Zadeh [8] ,it was followed up by Dubois and Prade [9], who used the extension principle in their approach. Other methods have been discussed by Puri and Ralescu [10] and Goetschel 
and Voxman [11].Fuzzy Differential Equations and initial value problems were regularly treated by O.Kaleva in [12] and S.Seikkala in [13].

The numerical methods for solving fuzzy differential equations are introduced in $[14,15,16, . .$.$] .$

Today, Fuzzy Differential Equations as a method of modelling dynamic system is considered.

Fuzzy Differential Equations with fuzzy coefficient or fuzzy initial conditions and control them, as a major problem in control theory is introduced. Transfer the original problem to a linear programming problem as an appropriate method to solve them and determine control function is presented.

For the first time, A.V.Kamyad(AVK) at el. Proposed an approach that may find optimal control for nonlinear problems with uncertain parameters (NPUP) and to solve an equivalent problem by a nonlinear programming. $[1,2,3,4,5]$

In this paper we proposed new approach to solve system of FDEs by LP problem[6,7]

In this approach we define an equivalent minimization problem for the FDEs system and substitute this system with an equivalent LP problem by discretizing.The Criterion function defined is trying in all of $\alpha$-level, solution obtained from optimization problem applied to original system.

In fact, this is the approximate solution of the original problem which is the best solution for the original FDEs in $L_{p}$ space. Moreover the error of this approximated solution is controllable. Finally our algorithm will be confirmed by simulation of different FDEs

\section{Definitions and AVK method}

Definition 1 : We focus on following problem

$$
x^{\prime}(t)=f(x(t), u(t), t)
$$

s.t $\quad x(a)=x_{a} x(b)=x_{b}$

where $f$ is a continuous function from $X \times U \times[a, b]$ to $R^{n}$, so that $x(t) \in X \subset R^{n}$ is state function, $u(t) \in U \subset R^{m}$ is control function and $t \in[a, b] \subseteq R$ is independent variable so called, time. $X$ and $U$ are compact subsets and must be chosen as the system reaches from initial state $x(a)$ to final state $x(b)$. We suppose the linear system (1)is stable in $[a, b]$ interval.

Definition 2 : First, consider system (1) we define following functional that is called the total error functional. Let

$$
E_{1}(x(t), u(t), t)=\int_{a}^{b}\|\dot{x}(t)-f(x(t), u(t), t)\| d t
$$

where $E_{1}: X \times U \times[a, b] \rightarrow R$ is a continuous functional. Now, the following key theorem is demonstrated. 
F. Nobakht, A.V. Kamyad, GH. Atazandi, A. Zare / TJMCS Vol .3 No.3 (2011) 346 - 356

Theorem 1 : If $\mathrm{h}$ is a nonlinear continuous function on $\mathrm{X} \times \mathrm{U} \times[\mathrm{a}, \mathrm{b}]$ and non-negative $(h \geq 0)$, then the necessary and sufficient condition for $\int_{a}^{b} h d x=0$ is $\mathrm{h} \equiv 0$ on $[\mathrm{a}, \mathrm{b}]$.

\section{Proof :[1]}

Theorem 2 : Necessary and sufficient condition for a function to be concluded a system (1) with initial condition $x(a)$ and final state $x(b)$ is to satisfy the following relation in problem(2):

$$
E_{1}(x(t), u(t), t)=0
$$

Proof : [1]

\subsection{AVK method}

Consider system of fuzzy differential equations given by

$$
\begin{aligned}
& \dot{x}(t)=f(x(t), u(t)) \\
& x(0)=x_{a} \\
& x(1)=x_{b}
\end{aligned}
$$

In AVK method, the following problem is defined in calculus of variations

$$
\begin{aligned}
& \underset{\substack{\text { Minimize } \\
x, u, t}}{ } E_{1}(x(t), u(t), t,)=\int_{0}^{1}(|\dot{x}(t)-f(x(t), u(t), t)|) d t \\
& \text { s.t } \quad x(0)=x_{a} \quad x(1)=x_{b}
\end{aligned}
$$

We assume the optimal solution of problem (5) is $x^{*}(t), u^{*}(t)$, the state and the control functions, respectively. And according to theorems 1,2 we have

$$
E_{1}\left(x^{*}(t), u^{*}(t), t\right)=0
$$

i.e.

$$
\begin{aligned}
& \dot{x}^{*}(t)=f\left(x^{*}(t), u^{*}(t), t\right) \quad t \in[0,1] \\
& x^{*}(0)=x_{a} \quad x^{*}(1)=x_{b}
\end{aligned}
$$

Then in general, for solving system (1) we can solve the minimization problem (5) by theorems 1 and 2. Thus the optimal solution of problem (1) is $x^{*}(t), u^{*}(t)$ from optimization problem (5) 


\section{Adams-bashforth method for solving system of fuzzy differential equations}

Consider the IVP of fuzzy differential equations given by

$$
\left\{\begin{array}{l}
x^{\prime}(t)=f(x, u, t) \\
x(0)=x_{0}, x(1)=x_{1}
\end{array}\right.
$$

In this section we use [2] to present Adams-bashforth methods for solving FIVP with fuzzy initial condition and fuzzy coefficient.

In this section we use [2] to present two step methods for solving FIVP with fuzzy initial condition and fuzzy coefficient.

Adams-bashforth methods is as follow:

$\left\{\begin{array}{l}\underline{x}\left(t_{i+1} ; \alpha\right)=\underline{x}\left(t_{i} ; \alpha\right)-\left(\frac{h}{2}\right) \bar{f}\left(t_{i-1}, x\left(t_{i-1}\right) ; \alpha\right)+\left(\frac{3 h}{2}\right) \underline{f}\left(t_{i}, x\left(t_{i}\right) ; \alpha\right) \\ \bar{x}\left(t_{i+1} ; \alpha\right)=\bar{x}\left(t_{i} ; \alpha\right)-\left(\frac{h}{2}\right) \underline{f}\left(t_{i-1}, x\left(t_{i-1}\right) ; \alpha\right)+\left(\frac{3 h}{2}\right) \bar{f}\left(t_{i}, x\left(t_{i}\right) ; \alpha\right) \\ i=1,2, \ldots, N-1, \\ 0 \leq \alpha \leq 1,\end{array}\right.$

\section{Optimal control for system of K-th order fuzzy differential equations}

Consider the system of fuzzy differential equations given by

$\left\{\begin{array}{l}x^{\prime}(t)=f(x, u, t) \\ x(0)=x_{0}, x(1)=x_{1}\end{array}\right.$

(10)

Where $x(t)$ is a fuzzy function of the crisp variable $t$.and $x^{\prime}(t)$ is fuzzy derivate of $x(t)$, and $f(x, u, t)$ is a fuzzy function of the crisp variable $t$ and fuzzy variable $x(t), u(t)$.we partition the interval $0 \leq \alpha \leq 1$ to $m$ equal part, where $m$ is arbitrary fixed positive integer:

$\alpha_{k}=k \frac{1-0}{m}$

Where $k=0, \ldots, m$ and $\alpha_{0}=0, \alpha_{m}=1$.then we have 
F. Nobakht, A.V. Kamyad, GH. Atazandi, A. Zare / TJMCS Vol .3 No.3 (2011) 346 - 356

$$
\left\{\begin{array}{l}
\underline{x}^{\prime \alpha_{0}}(t)=\underline{f}^{\alpha_{0}}(x, u, t) \\
{\underline{x^{\prime}}}^{\alpha_{1}}(t)=\underline{f}^{\alpha_{1}}(x, u, t) \\
\vdots \\
{\underline{x^{\prime}}}^{\alpha_{m}}(t)=\underline{f}^{\alpha_{m}}(x, u, t) \\
\bar{x}^{\alpha_{0}}(t)=\bar{f} \\
{\overline{x^{\prime}}}^{\alpha_{1}}(t)=\bar{f}(x, u, t) \\
\vdots \\
\bar{x}^{\alpha^{\prime}}(x, u, t) \\
\end{array}\right.
$$

The best solution for the optimization problem (11) is minimizing the total error of above system, i.e total error in $L_{1}$ space is minimized as follows:

$$
\begin{aligned}
& \text { Minimize } \\
& \int_{0}^{1}\left\{\sum_{k=0}^{m}\left[\left|\underline{\dot{x}}^{\alpha_{k}}(t)-\underline{f}^{\alpha_{k}}(x, u, t)\right|+\left|\overline{\dot{x}}^{\alpha_{k}}(t)-\bar{f}^{\alpha_{k}}(x, u, t)\right|\right]\right\} d t
\end{aligned}
$$

s.t

$$
\left\{\begin{array}{l}
\underline{x}^{\alpha_{k}}(0)=\underline{x}_{0}^{\alpha_{k}}, \underline{x}^{\alpha_{k}}(1)=\underline{x}_{1}^{\alpha_{k}} \\
\bar{x}^{\alpha_{k}}(0)=\bar{x}_{0}^{\alpha_{k}}, \bar{x}^{\alpha_{k}}(1)=\bar{x}_{1}^{\alpha_{k}}
\end{array}\right.
$$

Now we will solve problem (12) approximately

\section{Discretization}

We partition the interval $t \in[0,1]$ to $N$ equal subintervals (cells) and assume that $h=\frac{b-a}{N}$, where $N$ is arbitrary fixed positive integer. Then problem (12) yields to

$$
\text { Minimize } \quad \sum_{i=0}^{N-1} \int_{(i-1) / N}^{i / N}\left(\sum_{k=0}^{m}\left[\left|\underline{\dot{x}}^{\alpha_{k}}(t)-\underline{f}^{\alpha_{k}}(x, u, t)\right|+\left|\dot{x}^{\alpha_{k}}(t)-\bar{f}^{\alpha_{k}}(x, u, t)\right|\right] d t\right.
$$


Remark : As we know, an approximate value of integral $\int_{a}^{b} K(x) d x$ is $(b-a) K(c)$, where $c$ is any point such as $a \leq c \leq b$. So, applying above remark, and assume $c$ is an ending point in any subinterval, minimization problem (13) is formed as

$$
\underset{x, u}{\operatorname{Minimize}} \quad \sum_{i=0}^{N-1} \sum_{k=0}^{m} h\left(\left|\underline{\dot{x}}^{\alpha_{k}}(t)-\underline{f}^{\alpha_{k}}(x, u, t)\right|+\left|\overline{\dot{x}}^{\alpha_{k}}(t)-\bar{f}^{\alpha_{k}}(x, u, t)\right|\right)
$$

then by using Adams-bashforth method [2] we have

\section{$\underset{x, u}{\operatorname{Minimize}}$}

$$
\begin{aligned}
& \sum_{i=0}^{N-1} \sum_{K=0}^{m}[\overbrace{\left(\left|\underline{x}^{\alpha_{k}}\left(t_{i+1}\right)-\underline{x}^{\alpha_{k}}\left(t_{i}\right)-\frac{3 h}{2}\left(\underline{f}^{\alpha_{k}}\left(x\left(t_{i}\right), u\left(t_{i}\right) ; \alpha_{k}\right)\right)+\frac{h}{2}\left(\bar{f}^{\alpha_{k}}\left(x\left(t_{i-1}\right), u\left(t_{i-1}\right) ; \alpha_{k}\right)\right)\right|\right)}^{E_{1}} \\
& +\underbrace{\left(\mid-\alpha^{\alpha_{k}}\left(t_{i+1}\right)-x^{\alpha_{k}}\left(t_{i}\right)-\frac{3 h}{2}\left(\bar{f}^{\alpha_{k}}\left(x\left(t_{i}\right), u\left(t_{i}\right) ; \alpha_{k}\right)\right)+\frac{h}{2}\left(f^{\alpha_{k}}\left(x\left(t_{i-1}\right), u\left(t_{i-1}\right) ; \alpha_{k}\right)\right)\right)}_{E_{2}}] \times h
\end{aligned}
$$

$$
\text { s.t }\left\{\begin{array}{c}
\underline{x}^{\alpha_{k}}(0)=\underline{x}_{0}^{\alpha_{k}} \\
\underline{x}^{\alpha_{k}}(1)=\underline{x}_{1}^{\alpha_{k}} \\
\bar{x}^{\alpha_{k}}(0)=\bar{x}_{0}^{\alpha_{k}} \\
\bar{x}^{\alpha_{k}}(1)=\bar{x}^{\alpha_{k}} \\
\underline{x}^{\alpha_{k}}\left(t_{i}\right) \leq \bar{x}{ }^{\alpha_{k}}\left(t_{i}\right) \\
\underline{u}^{\alpha_{k}}\left(t_{i}\right) \leq \bar{u}^{\alpha_{k}}\left(t_{i}\right) \\
0 \leq \alpha \leq 1,0 \leq k \leq m
\end{array}\right.
$$

as a whole, problem (14) is a NLP problem and we may obtain its solution by many packages such as Matlab, Lingo,etc. since $f^{\alpha_{k}}(x, u, t)$ is a linear function, can transfer it to a linear programming problem (LPP)[9], so we may decompose the value of $E_{i}^{\alpha_{k}}$ by a difference of two nonnegative values $S_{1 i}^{\alpha_{k}}$ and $S_{2 i}^{\alpha_{k}}$,i.e, $S_{1 i}^{\alpha_{k}}, S_{2 i}^{\alpha_{k}} \geq 0$ as follow:

$E_{i}^{\alpha_{k}}=S_{1 i}^{\alpha_{k}}-S_{2 i}^{\alpha_{k}}$

Where $i=0, \ldots, N-1$ and $k=0, \ldots, m$.consider the determination of absolute value in definition of norm function, so that

$\left|E_{i}^{\alpha_{k}}\right|=S_{1 i}^{\alpha_{k}}+S_{2 i}^{\alpha_{k}}$

Then we have 


$$
\begin{aligned}
& \left|\underline{x}^{\alpha_{k}}\left(t_{i+1}\right)-\underline{x}^{\alpha_{k}}\left(t_{i}\right)-\frac{3 h}{2}\left(\underline{f}^{\alpha_{k}}\left(x\left(t_{i}\right), u\left(t_{i}\right) ; \alpha_{k}\right)\right)+\frac{h}{2}\left(\bar{f}^{\alpha_{k}}\left(x\left(t_{i-1}\right), u\left(t_{i-1}\right) ; \alpha_{k}\right)\right)\right| \\
& =y_{i}^{\alpha_{k}}+v_{i}^{\alpha_{k}} \\
& \underline{x}^{\alpha_{k}}\left(t_{i+1}\right)-\underline{x}^{\alpha_{k}}\left(t_{i}\right)-\frac{3 h}{2}\left(\underline{f}^{\alpha_{k}}\left(x\left(t_{i}\right), u\left(t_{i}\right) ; \alpha_{k}\right)\right)+\frac{h}{2}\left(\bar{f}^{\alpha_{k}}\left(x\left(t_{i-1}\right), u\left(t_{i-1}\right) ; \alpha_{k}\right)\right) \\
& =y_{i}^{\alpha_{k}}-v_{i}^{\alpha_{k}} \\
& \left|-\alpha^{\alpha_{k}}\left(t_{i+1}\right)-\bar{x}^{\alpha_{k}}\left(t_{i}\right)-\frac{3 h}{2}\left(\bar{f}^{\alpha_{k}}\left(x\left(t_{i}\right), u\left(t_{i}\right) ; \alpha_{k}\right)\right)+\frac{h}{2}\left(\underline{f}^{\alpha_{k}}\left(x\left(t_{i-1}\right), u\left(t_{i-1}\right) ; \alpha_{k}\right)\right)\right| \\
& =z_{i}^{\alpha_{k}}+w_{i}^{\alpha_{k}} \\
& -\alpha^{\alpha_{k}}\left(t_{i+1}\right)-\bar{x}^{\alpha_{k}}\left(t_{i}\right)-\frac{3 h}{2}\left(\bar{f}^{\alpha_{k}}\left(x\left(t_{i}\right), u\left(t_{i}\right) ; \alpha_{k}\right)\right)+\frac{h}{2}\left(\underline{f}^{\alpha_{k}}\left(x\left(t_{i-1}\right), u\left(t_{i-1}\right) ; \alpha_{k}\right)\right) \\
& =z_{i}^{\alpha_{k}}-w_{i}^{\alpha_{k}} \\
& k=0, \ldots, m, i=0, \ldots, N-1,0 \leq \alpha \leq 1 \\
& y_{i}^{\alpha_{k}}, v_{i}^{\alpha_{k}}, z_{i}^{\alpha_{k}}, w_{i}^{\alpha_{k}} \geq 0
\end{aligned}
$$

At the end ,the minimization problem (14) is formed as below LPP

$$
\underset{x, u}{\operatorname{Minimize}} \sum_{i=0}^{N-1} \sum_{k=0}^{m}\left\{y_{i}^{\alpha_{k}}+v_{i}^{\alpha_{k}}+z_{i}^{\alpha_{k}}+w_{i}^{\alpha_{k}}\right\} \times h
$$

s.t

$$
\begin{aligned}
& \left\{\begin{array}{l}
\underline{x}^{\alpha_{k}}\left(t_{i+1}\right)-\underline{x}^{\alpha_{k}}\left(t_{i}\right)-\frac{3 h}{2}\left(\underline{f}^{\alpha_{k}}\left(x\left(t_{i}\right), u\left(t_{i}\right) ; \alpha_{k}\right)\right)+\frac{h}{2}\left(\bar{f}^{\alpha_{k}}\left(x\left(t_{i-1}\right), u\left(t_{i-1}\right) ; \alpha_{k}\right)\right) \\
=y_{i}^{\alpha_{k}}-v_{i}^{\alpha_{k}} \\
\bar{x}^{\alpha_{k}}\left(t_{i+1}\right)-\bar{x}^{\alpha_{k}}\left(t_{i}\right)-\frac{3 h}{2}\left(\bar{f}^{\alpha_{k}}\left(x\left(t_{i}\right), u\left(t_{i}\right) ; \alpha_{k}\right)\right)+\frac{h}{2}\left(\underline{f}^{\alpha_{k}}\left(x\left(t_{i-1}\right), u\left(t_{i-1}\right) ; \alpha_{k}\right)\right) \\
=z_{i}^{\alpha_{k}}-w_{i}^{\alpha_{k}}
\end{array}\right. \\
& \left\{\begin{array}{l}
\underline{x}^{\alpha_{k}}(0)=\underline{x}_{0}^{\alpha_{k}}, \underline{x}^{\alpha_{k}}(1)=\underline{x}^{\alpha_{k}} \\
\bar{x}^{\alpha_{k}}(0)=\bar{x}^{\alpha_{k}}, \bar{x}^{\alpha_{k}}(1)=\bar{x}_{1}{ }^{\alpha_{k}} \\
\underline{x}^{\alpha_{k}}\left(t_{i}\right) \leq \bar{x}^{\alpha_{k}}\left(t_{i}\right), \underline{u}^{\alpha_{k}}\left(t_{i}\right) \leq \bar{u}^{\alpha_{k}}\left(t_{i}\right), \\
\underline{x}^{\alpha_{k}}\left(t_{i}\right) \leq \underline{x}^{\alpha_{k+1}}\left(t_{i}\right), \underline{u}^{\alpha_{k}}\left(t_{i}\right) \leq \underline{u}^{\alpha_{k+1}}\left(t_{i}\right), \\
\bar{x}^{\alpha_{k+1}}\left(t_{i}\right) \leq \bar{x}^{\alpha_{k}}\left(t_{i}\right), \bar{u}^{\alpha_{k+1}}\left(t_{i}\right) \leq \bar{u}^{\alpha_{k}}\left(t_{i}\right) \\
y_{i}^{\alpha_{k}}, v_{i}^{\alpha_{k}}, z_{i}^{\alpha_{k}}, w_{i}^{\alpha_{k}} \geq 0 \\
k=0, \ldots, m, i=0, \ldots, N-1,0 \leq \alpha \leq 1
\end{array}\right.
\end{aligned}
$$

Then non linear programming (10) translate to linear programming (16) 
F. Nobakht, A.V. Kamyad, GH. Atazandi, A. Zare / TJMCS Vol .3 No.3 (2011) 346 - 356

Finally by obtaining the solution of problem (16) we recognize the value of unknown admissible pair $\left(x_{i}, u_{i}\right)$ state and control function .We can construct the optimal solution for FIVP (1) by two functions $\left(x^{*}{ }_{i}, u^{*}{ }_{i}\right)$.

\section{Example}

In this section, we use our algorithm for fuzzy differential equation with fuzzy parameter and fuzzy initial value, Consider the fuzzy system given by

$$
\left\{\begin{array}{l}
\tilde{y}^{\prime \prime}(x)-\tilde{a} \tilde{y}^{\prime}(x)-\tilde{b} \tilde{y}(x)-\tilde{c} \tilde{u}(x)=0 \\
\tilde{a}(r)=(0.1+0.01 r, 0.12-0.01 r) \\
\tilde{b}(r)=(1+0.01 r, 1.03-0.02 r) \\
\tilde{c}(r)=(0.3+0.01 r, 0.32-0.01 r) \\
y(0 ; r)=(0.01+0.01 r, 0.021-0.001 r) \\
y^{\prime}(0 ; r)=(3+.1 r, 3.2-0.1 r) \\
y(1 ; r)=(4+0.5 r, 5-0.5 r) \\
y^{\prime}(1 ; r)=(6.2+0.1 r, 7.4-1.1 r) \\
0 \leq r \leq 1
\end{array}\right.
$$

In order to obtain optimal control of FDEs (17), suppose that

$$
\left\{\begin{array}{l}
y_{1}(x)=y(x) \\
y_{2}(x)=y^{\prime}(x)
\end{array}\right.
$$

Then we transformed this second order FDEs (17) to the following system of first order FDEs

$$
\left\{\begin{array}{l}
\tilde{y}_{1}^{\prime}=\tilde{y}_{2} \\
\tilde{y}_{2}^{\prime}=\tilde{b} \tilde{y}_{1}+\tilde{a} \tilde{y}_{2}+\tilde{c} \tilde{u}
\end{array}\right.
$$

Now by using $\alpha$-level sets we have 
F. Nobakht, A.V. Kamyad, GH. Atazandi, A. Zare / TJMCS Vol .3 №.3 (2011) 346 - 356

$$
\left\{\begin{array}{l}
\underline{y}_{1}^{\prime}(x ; \alpha)=\underline{y}_{2}(x ; \alpha) \\
\underline{y}_{2}^{\prime}(x ; \alpha)=\underline{b(\alpha) y_{1}(x ; \alpha)+a(\alpha) y_{2}(x ; \alpha)+c(\alpha) u(x ; \alpha)} \\
\underline{y}_{1}(0 ; \alpha)=(0.01+0.01 \alpha), \\
\underline{y}_{2}(0 ; \alpha)=(3+0.1 \alpha), \\
\underline{y}_{1}(1 ; \alpha)=(4+0.5 \alpha), \\
\underline{y}_{2}(1 ; \alpha)=(6.2+0.1 \alpha), \\
\bar{y}_{1}^{\prime}(x ; \alpha)=\bar{y}_{2}(x ; \alpha) \\
\bar{y}_{2}^{\prime}(x ; \alpha)=\overline{b(\alpha) y_{1}(x ; \alpha)+a(\alpha) y_{2}(x ; \alpha)+c(\alpha) u(x ; \alpha)} \\
\bar{y}_{1}(0 ; \alpha)=(0.021-0.001 \alpha), \\
\bar{y}_{2}(0 ; \alpha)=(3.2-0.1 \alpha), \\
\bar{y}_{1}(1 ; \alpha)=(5-0.5 \alpha), \\
\bar{y}_{2}(1 ; \alpha)=(7.4-1.1 \alpha),
\end{array}\right.
$$

For some values of $\alpha, 0 \leq \alpha \leq 1$ the state and control function of(17) with $\mathrm{N}=5$ and $\mathrm{m}=5$ are given in Figure(1) and Figure(2)
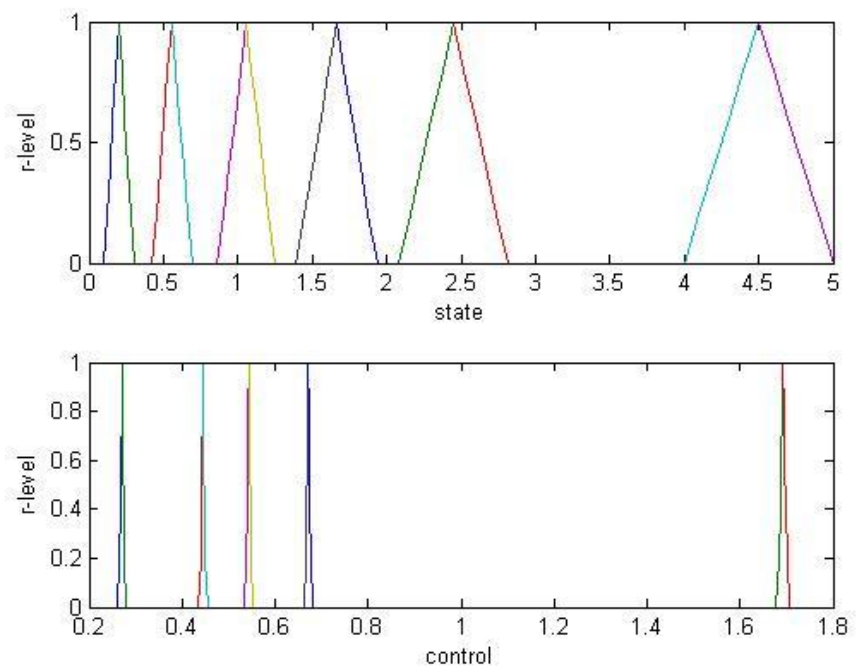

Figure 1:state and control function 
F. Nobakht, A.V. Kamyad, GH. Atazandi, A. Zare / TJMCS Vol .3 No.3 (2011) 346 - 356
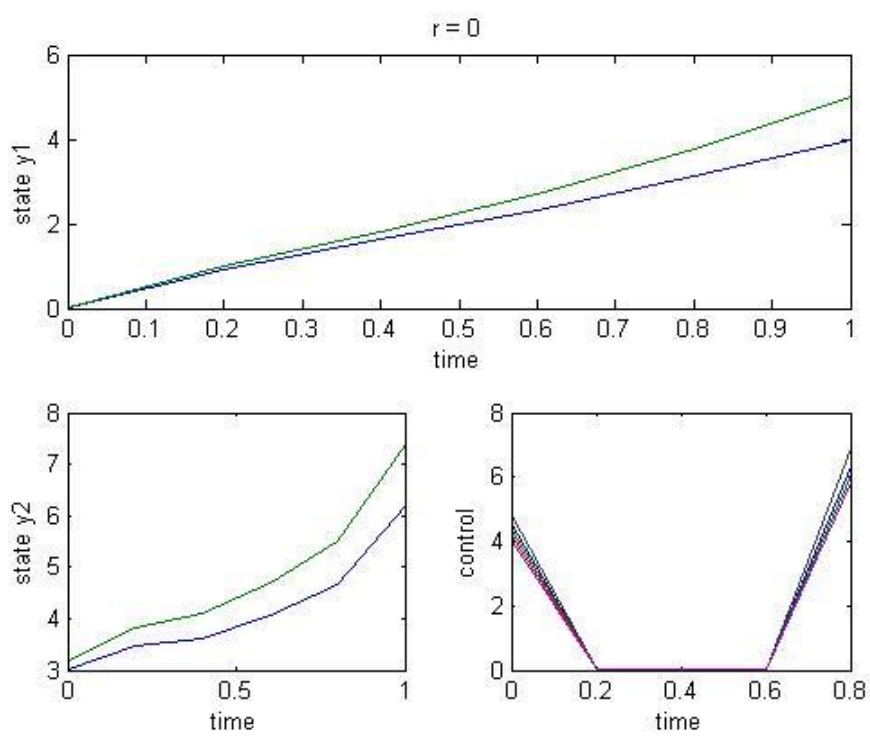

Figure 2:state and control function

the value of total error functional for $N=10$ and $m=5$ is $E=0.06400$, thus the state and control function are optimal solution problem (17).

1] K.P. Badakhshan, A.V. Kamyad, A. Azemi,using AVK method to solve nonlinear problems with uncertain parametes,isse1,volume 189,1jun2007

[2] T. Allahviranloo, N. Ahmady, E. Ahmady, Two step method for fuzzy differentional equations, International mathematical forum ,1,2006,no.17,823-832

[3] M. Ma , M. Friedman, A. Kandel, numerical solution of fuzzy differential equations, fuzzy set and system105(1999) 113-138

[4] J.J. Buckley and E. Eslami, introduction to fuzzy logic and fuzzy sets, physicaverlag,Heidelberg,germany,2001

[5] S. Seikkala, on the fuzzy initial value problem, fuzzy sets and systems, 24(1987),pp.319

[6] A. Heydari, A.V. Kamyad and M.H. Farahi, A new approach for solving robust control problem, J. Inst. Math.Comput. Sci. 15 (2002) (1), pp. 1-7.

[7] A. Zare, A.K. Sedigh and A.V. Kamyad, Applied measure theory in robust tracking, Mag. EECI 1 (2002)(1), pp. 11-17.

[8] S.L. Chang and L.A. Zadeh, On fuzzy mapping and control, IEEE Trans. System Man Cybernet, 2(1972), pp . 30-34 
F. Nobakht, A.V. Kamyad, GH. Atazandi, A. Zare / TJMCS Vol .3 №.3 (2011) 346 - 356

[9] D. Dubois , H. Prade , Towards fuzzy differential calculus: part 3, differentiation, Fuzzy sets and systems .8(1982) 225-233

[10] M.L. Puri , D.A. Ralescu, Differentials of fuzzy functions,J.Math.Analysis.Appl.91(1983)

[12] O. Kaleva, The Cauchy problem for fuzzy differential equations, J.Fuzzy sets and systems 35(1990)389-396

[13] S. Seikkala, On the fuzzy initial value problem, J. Fuzzy sets and systems, 24(1987),pp.319-330

[14] M. Friedman, M. Ma, A. Kandel, Numerical solution of fuzzy differential and integral equations, J. Fuzzy sets and systems, Vol.106, pp.35-48,1999

[15] M. Ma, M. Frriedman, A. Kandel, Numerical solution of fuzzy differential equations, J. Fuzzy sets and systems 105(1999)133-138

[16] T. Allahviranloo, N. Ahmady, E. Ahmady, Two step method for fuzzy differential equatios, International Mathematical Forum, 1, 2006,no. 17,823-832 